\title{
Network pharmacology based study of LW-AFC, a new formula from a traditional Chinese medicinal prescription, on neuroendocrine immunomodulation network
}

\author{
Wenxia Zhou, Jianhui Wang, Yan Huang, Lu Han, Xiaorui Cheng, Yongxiang Zhang \\ Beijing Institute of Pharmacology and Toxicology, China
}

Alzheimer's disease (AD) is the leading cause of dementia that affects millions of elderly people worldwide. The currently available therapies have limited efficacy. Liuwei Dihuang decoction (LW), a classical traditional Chinese medicine (TCM) formula, has long been used to treat various diseases, including dementia. A large number of pharmacological studies have showed that $\mathrm{LW}$ has beneficial effects on $\mathrm{AD}$. LW-AFC is a new formula consisted of the main active components prepared from LW. In this study, the effects of LW-AFC on AD mouse model were investigated, and its pharmacological mechanisms were also studied based on network pharmacological strategy. The results showed that administration of LWAFC significantly improved behavioral performances in spontaneous locomotor activity, object recognition memory, spatial learning and memory, passive and active avoidance impairment in some AD mouse models, such as SAMP8 and APP/PS1 transgenic mice. Meanwhile, the impairments of long-term potentiation (LTP) were significantly ameliorated by LW-AFC administration in SAMP8 mice, as well as in corticosterone- induced LTP inhibition mice model. These effects were companied by alleviating of the neuron loss in the hippocampus, suppressing of A-beta deposition in the brain, and reducing of the concentration of Abeta42 in the hippocampus and plasma of APP/PS1 mice. The restoring and correcting of imbalance of hypothalamic-pituitary-adrenal (HPA) and hypothalamic-pituitary-gonadal (HPG) axis, the disorder of lymphocyte subsets, the abnormality of production of cytokine were also observed in LW-AFC treated SAMP8 and APP/PS1 mice. The findings in this study indicated that LW-AFC ameliorated the behavioral and pathological deterioration of $\mathrm{AD}$ mice via the restoration of neuroendocrine immunomodulation (NIM) network in a holistic way, suggesting that LW-AFC might be a promising therapy for AD. 